\title{
Comparative analysis of microsatellite and SNP markers for parentage testing in the golden snub-nosed monkey (Rhinopithecus roxellana)
}

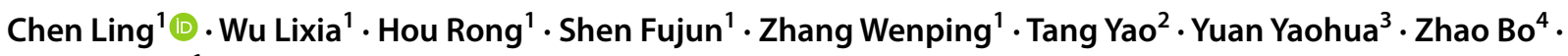 \\ Zhang Liang'
}

Received: 13 November 2019 / Accepted: 30 March 2020 / Published online: 3 April 2020

(c) The Author(s) 2020

\begin{abstract}
Microsatellite markers are popular for assigning parentage, but single-nucleotide polymorphisms (SNPs) have only been applied in this area recently. To evaluate these two markers which have been previously studied in golden snub-nosed monkeys, we genotyped 12 individuals using 37 microsatellite loci and 37 SNP markers. The data showed that 32 of 37 microsatellite loci were polymorphic, and most microsatellite loci were high informative (mean PIC $=0.599$ ). Meanwhile, 24 of 37 SNP markers were polymorphic and most were low informative (mean PIC $=0.244$ ). For microsatellites, the combined exclusion probability with one-parent-unknown/known (CE-1P/CE-2P) nearly reached 1, while for the SNP markers, CE-2P only reached 0.9582 . Under the condition of one parent known/unknown, the CE-2P and CE-1P could meet the international human parental standard (0.9973) by using five or nine microsatellite loci respectively. For SNP markers, we doubled the loci $(n=48)$ and simulated parentage testing, and the data showed that the CE-2P was 0.998 while the CE-1P was still low. This result indicated that the SNP loci which we used here had low polymorphism and that more loci need to be developed in the future. In addition, we corrected one case of failed identification by excluding siblings and reducing the range of candidate paternities.
\end{abstract}

Keywords Rhinopithecus roxellana $\cdot$ microsatellite $\cdot$ SNP $\cdot$ parentage testing

\section{Introduction}

The golden snub-nosed monkey is an endemic endangered species in China with a current wild population of $~ 22,000$ individuals. According to data from the 2017 International Studbook of Golden Monkey (Chinese Association of Zoological Gardens), at the end of 2017, there were 488 individuals in captivity in 46 institutes around the world. Genetic management is essential for the conservation of both the exsitu and in-situ populations of endangered wildlife. Although

Zhang Liang

lianne@panda.org.cn

1 Chengdu Research Base of Giant Panda Breeding, Sichuan Key Laboratory of Conservation Biology for Endangered Wildlife, Sichuan Academy of Giant Panda, Chengdu 610057, Sichuan, China

2 Fuzhou Zoo, Fuzhou 350000, Fujian, China

3 Shanghai Zoo, Shanghai 200336, China

4 Chengdu Zoo, Sichuan 610081, China much research has been done on the population structure and population dynamics of this species, parentage testing has not developed well.

In captivity, revising the pedigree by parentage testing can help to reduce problems caused by inbreeding such as low viability, low fertility, and high mortality. Microsatellite markers have been used in the animal husbandry industry, initially for horses, since the 1990s because of their high rate of polymorphism and low required marker numbers. Since then, they have been successfully used in many threatened animals such as Ailuropoda melanoleuca (Zhang et al. 2003), Panthera tigris amoyensis (Zhang et al. 2006), and Grus japonensis (Zhang et al. 2015). Single Nucleotide Polymorphisms (SNPs), a third-generation genetic marker, has the advantages of good genetic stability, rapid detection, high multi-density, and wide distribution, and has been applied to the animal kingdom, and especially the animal husbandry industry, for parentage testing (Heaton et al. 2002; Werner et al. 2004).

Microsatellites have historically been preferred in many evolution studies of golden snub-nosed monkeys, while 
SNPs have only been applied to this area recently. Microsatellites of primates such as Homo sapiens, Cebus paella and Macaca mulatta have been used in studies of the golden snub-nosed monkeys before specific markers for this species were published (Pan et al. 2005; Ren 2007; He 2013). Twelve years ago, the first 11 microsatellite loci of the golden snub-nosed monkey were isolated (Hao et al. 2007). Ren 2007)conducted a parentage testing for five captive golden snub-nosed monkeys in Xi' an, and Zhou et al. 2015) used 11 loci to perform parentage testing on Shennongjia golden monkeys. However, none of the CE-1P and CE-2P reached the international parental standard. In 2015, 37 SNP loci were developed from the golden snub-nosed monkey genome (Du 2015). High-resolution dissolution curve (HRM) technology has been employed on seven individuals for SNP typing and individual identification without parentage testing.

To compare the difference in accuracy of parentage testing between microsatellites and SNP markers on golden snub-nosed monkeys, we utilized the above-mentioned markers for parentage testing of 12 individuals. This research can be used to help in field surveys and genetic management of captive populations.

\section{Materials and methods}

\section{Experimental and materials}

Blood samples from Fuzhou Zoo $(\mathrm{n}=5)$ and Shanghai Zoo $(n=6)$, a tissue sample from the Gene Resource Library of Chengdu Research Base of Giant Panda Breeding $(n=1)$, and fresh feces samples from Chengdu Zoo $(n=17)$ were used to parentage test golden snub-nosed monkeys using the 37 published microsatellite loci and the 37 SNP loci. The kinship of the above 12 individuals from which the blood and tissue samples were collected was clearly recorded
(Table 1). All sample collection and utility protocols in this study were approved by the Chengdu Research Base of Giant Panda Breeding. The experimental procedures were fully in compliance with the current laws on animal welfare and research in China.

\section{DNA extraction and quality determination}

DNA was extracted using the QIAamp DNA Blood Midi Kit (Qiagen, Germany) for blood samples, the DNeasy Blood and Tissue Kit (Qiagen, Germany) for tissue samples, and the QIAamp Fast DNA Stool Mini Kit (Qiagen, Germany) for feces samples, according to the manufacturer's protocol. The DNA concentration in the samples was determined using a NanoDrop 2000 spectrophotometer (Thermo Scientific, USA) at an optical density (OD value) of $260 \mathrm{~nm}$; an OD260:OD280 ratio ranging between 1.8 and 2.0 was considered acceptable.

\section{Microsatellite loci isolation PCR amplification}

A total of 37 loci were synthesized for PCR amplification with reference to relevant literature (Pan et al. 2005; Haoet al. 2007; Ren 2007; He 2013). The reaction mixture contained 10-20 ng DNA, $1 \mu \mathrm{L} 10 \times$ Taq buffer, $0.2 \mu \mathrm{L}$ dNTP Mix, $0.1 \mu \mathrm{L}$ Taq DNA polymerase, $0.2 \mu \mathrm{L}$ each primer, 0.8 $\mu \mathrm{L} \mathrm{MgCl}_{2}$, and adjusted with RNase-free water to a final volume of $10 \mu \mathrm{L}$. The amplification reaction was performed by denaturing at $95^{\circ} \mathrm{C}$ for $10 \mathrm{~min}$; followed by 35 cycles of denaturing at $95^{\circ} \mathrm{C}$ for $30 \mathrm{~s}$, annealing temperature for $45 \mathrm{~s}$, and extending at $72{ }^{\circ} \mathrm{C}$ for $45 \mathrm{~s}$ and a final extension at $72{ }^{\circ} \mathrm{C}$ for $10 \mathrm{~min}$. The PCR products were detected by $2 \%$ agarose gel electrophoresis, and 37 loci were successfully amplified (Table 2). The PCR product was sent to Shanghai Sangon Bioengineering Co., Ltd. (Shanghai, China) for genotyping. The same operations were performed on the stool samples.
Table 1 Sample information of 12 golden monkey

\begin{tabular}{llllll}
\hline Sample name & Pedigree number & Sex & Age & Source & Type \\
\hline GMFZ01 & 480 & F & 12 & Fuzhou zoo & Blood \\
GMFZ02 & 483 & M & 12 & Fuzhou zoo & Blood \\
GMFZ03 & 577 & M & 8 & Fuzhou zoo & Blood \\
GMFZ04 & 834 & M & 1.5 & Fuzhou zoo & Blood \\
GMFZ05 & 799 & F & 7 & Fuzhou zoo & Blood \\
GM6 & UNK & UNK & UNK & Chengdu zoo & Cardiac muscle \\
GMSH07 & 223 & M & 19 & Shanghai zoo & Blood \\
GMSH08 & 384 & F & 13 & Shanghai zoo & Blood \\
GMSH09 & 790 & M & 4 & Shanghai zoo & Blood \\
GMSH10 & 183 & M & 23 & Shanghai zoo & Blood \\
GMSH11 & 356 & F & 14 & Shanghai zoo & Blood \\
GMSH12 & 835 & M & 3 & Shanghai zoo & Blood \\
\hline
\end{tabular}


Table 2 Microsatellite loci information used in the research

\begin{tabular}{|c|c|c|c|c|c|}
\hline Loci & Primer sequence $\left(5^{\prime}-3^{\prime}\right)$ & Repeat motif & Size range (bp) & $\operatorname{Tm}\left({ }^{\circ} \mathrm{C}\right)$ & References \\
\hline GM209 & $\begin{array}{l}\text { F:ATCTGAATGATGTGTGGATGT } \\
\text { R:TAGAGTAGCATTGCCT }\end{array}$ & Di & $146-158$ & 53 & Hao et al. (2007) \\
\hline GM219 & $\begin{array}{l}\text { F:GTGTATTGTGGGGCTATC } \\
\text { R:GTGGGCTCTGACCTAGGAATC }\end{array}$ & Di & $183-187$ & 53 & Hao et al. (2007) \\
\hline GM108 & $\begin{array}{l}\text { F:CAGCGTAAGCCAGTTGCC } \\
\text { R:GGAAAAGTCTGAAACCCACGA }\end{array}$ & Di & $123-137$ & 53 & Hao et al. (2007) \\
\hline GM228 & $\begin{array}{l}\text { F:ACCAGCCTCCAAAATTATGTG } \\
\text { R:GAGGGGTGACTGAGTCAAA }\end{array}$ & Di & $165-175$ & 52 & Hao et al. (2007) \\
\hline GM213 & $\begin{array}{l}\text { F:GCCCTAGCAGAACATGACACT } \\
\text { R:AGCCCATGCGTATTGAGT }\end{array}$ & Di & $182-198$ & 55 & Hao et al. (2007) \\
\hline GM105 & $\begin{array}{l}\text { F:CGGATCATTGTTGCTC } \\
\text { R:AGATGGGAAGGTGTGTCTACA }\end{array}$ & Di & $161-175$ & 55 & Hao et al. (2007) \\
\hline GM227 & $\begin{array}{l}\text { F:CAGAAGCCACCGAAATG } \\
\text { R:AATTCTCTCCCAAGGAATATG }\end{array}$ & Di & $148-174$ & 52 & Hao et al. (2007) \\
\hline GM214 & $\begin{array}{l}\text { F:GGGCAACAGAGCGAGACTG } \\
\text { R:TGCAAAGATGTGAACGGAAAT }\end{array}$ & Di & $134-154$ & 53 & Hao et al. (2007) \\
\hline GM206 & $\begin{array}{l}\text { F:GGTGCTACCAGATCATTGTT } \\
\text { R:CAGATGGGAAGGTGTGTCTAC }\end{array}$ & Di & $163-183$ & 54 & Hao et al. (2007) \\
\hline GM109 & $\begin{array}{l}\text { F:GGTGGAGGAGGGCCTAAC } \\
\text { R:CTGATGTCCATAGGCGACCAT }\end{array}$ & Di & $138-164$ & 54 & Hao et al. (2007) \\
\hline GM220 & $\begin{array}{l}\text { F:CCCTTCTCTGTGACCTTGT } \\
\text { R:TGAGTCCTCAGACACCAATCA }\end{array}$ & Di & $165-179$ & 52 & Hao et al. (2007) \\
\hline D20s206 & $\begin{array}{l}\text { F:TCCATTATTCCCCTCAAACA } \\
\text { R:GGTTTGCCATTCAGTTGAGA }\end{array}$ & Tetra & $163-164$ & 52 & He (2013) \\
\hline D5s 1457 & $\begin{array}{l}\text { F:TAGGTTCTGGGCATGTCTGT } \\
\text { R:TGCTTGGCACACTTCAGG }\end{array}$ & Tetra & $97-127$ & 57 & He (2013) \\
\hline D7s2204 & $\begin{array}{l}\text { F:TCATGACAAAACAGAAATTAAGTG } \\
\text { R:AGTAAATGGAATTGCTTGTTACC }\end{array}$ & Tetra & $217-269$ & 54 & $\mathrm{He}(2013)$ \\
\hline D14s306 & $\begin{array}{l}\text { F:AAAGCTACATCCAAATTAGGTAGG } \\
\text { R:TGACAAAGAAACTAAAATGTCCC }\end{array}$ & Tetra & $190-210$ & 52 & $\mathrm{He}(2013)$ \\
\hline D1s533 & $\begin{array}{l}\text { F:CATCCCCCCCAAAAAATATA } \\
\text { R:TTGCTAATCAAATAACAATGGG }\end{array}$ & Tetra & $193-225$ & 52 & Pan et al. (2005) \\
\hline D3s1768 & $\begin{array}{l}\text { F:GGTTGCTGCCAAAGATTAGA } \\
\text { R:CACTGTGATTTGCTGTTGGA }\end{array}$ & Tetra & 186-206 & 48 & Pan et al. (2005) \\
\hline D6s474 & $\begin{array}{l}\text { F:TGTACAAAAGCCTATTTAGTCAGG } \\
\text { R:TCATGTGAGCCAATTCCTCT }\end{array}$ & Tetra & $151-167$ & 58 & Pan et al. (2005) \\
\hline D6s493 & $\begin{array}{l}\text { F:TCATGACAAAACAGAAATTAAGTG } \\
\text { R:AGTAAATGGAATTGCTTGTTACC }\end{array}$ & Tetra & $236-237$ & 54 & Pan et al. (2005) \\
\hline D7s1830 & $\begin{array}{l}\text { F:GTACATGATGGGCTGTCCTC } \\
\text { R:GATACATACTGCCAATAAATCACA }\end{array}$ & Tetra & $200-228$ & 58 & Pan et al. (2005) \\
\hline D10s1432 & $\begin{array}{l}\text { F:CAGTGGACACTAAACACAATCC } \\
\text { R:TAGATTATCTAAATGGTGGATTTCC }\end{array}$ & Tetra & $157-185$ & 54 & Pan et al. (2005) \\
\hline D11s1366 & $\begin{array}{l}\text { F:GCTACAATGATAGGGAAATAATAGA } \\
\text { R:GGTGGGATCCTTTGCTATTT }\end{array}$ & Tetra & $241-242$ & 54 & Pan et al. (2005) \\
\hline D11s2002 & $\begin{array}{l}\text { F:CATGGCCCTTCTTTTCATAG } \\
\text { R:AATGAGGTCTTACTTTGTTGC }\end{array}$ & Tetra & $224-252$ & 58 & Pan et al. (2005) \\
\hline D15s644 & $\begin{array}{l}\text { F:CCTTCATTGGCAGACTCACT } \\
\text { R:GCAGACACCAAGATGATAACG }\end{array}$ & Tetra & 203-204 & 48 & Pan et al. (2005) \\
\hline D17s1290 & $\begin{array}{l}\text { F:GCCAACAGAGCAAGACTGTC } \\
\text { R:GGAAACAGTTAAATGGCCAA }\end{array}$ & Tetra & $170-210$ & 60 & Pan et al. (2005) \\
\hline
\end{tabular}


Table 2 (continued)

\begin{tabular}{|c|c|c|c|c|c|}
\hline Loci & Primer sequence $\left(5^{\prime}-3^{\prime}\right)$ & Repeat motif & Size range (bp) & $\operatorname{Tm}\left({ }^{\circ} \mathrm{C}\right)$ & References \\
\hline D18s851 & $\begin{array}{l}\text { F:CTGTCCTCTAGGCTCATTTAGC } \\
\text { R:TTATGAAGCAGTGATGCCAA }\end{array}$ & Tetra & $253-254$ & 50 & Pan et al. (2005) \\
\hline D11s925 & $\begin{array}{l}\text { F:AGAACCAAGGTCGTAAGTCCTG } \\
\text { R:TTAGACCATTATGGGGGCAA }\end{array}$ & Di & $172-199$ & 55 & Ren (2007) \\
\hline D13s 159 & $\begin{array}{l}\text { F:AGGCTGTGACTTTTAGGCCA } \\
\text { R:CCAGGCCACTTTTGATCTGT }\end{array}$ & Di & $168-203$ & 55 & Ren (2007) \\
\hline $\mathrm{D} 16 \mathrm{~s} 420$ & $\begin{array}{l}\text { F:ACAAGGGTTATGGGAGGTATGAG } \\
\text { R:GGCAATATAGTGAGAATACCAGTAG }\end{array}$ & Di & 248 & 54 & Ren (2007) \\
\hline D2s141 & $\begin{array}{l}\text { F:ACTAATTACTACCCNCACTCCC } \\
\text { R:TTTTCCAAACAGATACAGTGAACTT }\end{array}$ & Di & $152-178$ & 56 & Ren (2007) \\
\hline D6s271 & $\begin{array}{l}\text { F:AACAATTGGGAAATGGCTTA } \\
\text { R:TAGGTTGTGGTGGGTGTTAC }\end{array}$ & Di & $166-208$ & 55 & Ren (2007) \\
\hline D6s287 & $\begin{array}{l}\text { F:GCCTACAAGGATTTCCAAAT } \\
\text { R:AATGAGTGATAGCCTACAATGC }\end{array}$ & Di & 286 & 50 & Ren (2007) \\
\hline $\mathrm{D} 7 \mathrm{~s} 503$ & $\begin{array}{l}\text { F:ACTTGGAGTAATGGGAGCAG } \\
\text { R:GTCCCTGAAAACCTTTAATCAG }\end{array}$ & Di & $148-180$ & 55 & Ren (2007) \\
\hline D16S539 & $\begin{array}{l}\text { F:GATCCCAAGCTCTTCCTCTT } \\
\text { R:ACGTTTGTGTGTGCATCTGT }\end{array}$ & Tetra & $148-172$ & 55 & Ren (2007) \\
\hline D17S791 & $\begin{array}{l}\text { F:GTTTTCTCCAGTTATTCCCC } \\
\text { R:GCTCGTCCTTTGGAAGAGTT }\end{array}$ & Di & $165-199$ & 55 & Ren (2007) \\
\hline D4S431 & $\begin{array}{l}\text { F:AGGCATACTAGGCCGTATT } \\
\text { R:TTCCCATCAGCGTCTTC }\end{array}$ & Di & $246-270$ & 55 & Ren (2007) \\
\hline $\mathrm{D} 7 \mathrm{~s} 1826$ & $\begin{array}{l}\text { F:CATCCATCTATCTCTGTAАTCTCTC } \\
\text { R:TATTTAACACACCTGTCTCAАTCC }\end{array}$ & Tetra & $142-162$ & 54 & Ren (2007) \\
\hline
\end{tabular}

\section{SNP loci selection}

Referring to the study of Du 2015) 37 SNP loci were selected (Table 3). The primers were synthesized by Shanghai Sangon Bioengineering Co., Ltd. (Shanghai, China).

\section{HRM reaction and genotyping}

HRM genotyping was performed on Roche LightCycler 96. The reaction mixture contained $10 \mathrm{ng}$ DNA, $10 \mu \mathrm{L}$ HRM Master Mix (Roche, Germany), 0.4 $\mu \mathrm{L}$ of each primer, $1.6 \mu \mathrm{L} \mathrm{Mgcl}$ and adjusted with RNase-free water to a final volume of $20 \mu \mathrm{L}$. A negative control, which contained sterile water in place of template DNA, was added during each run of the PCR to check for contamination. The amplification was achieved by the following protocol: an initial denaturation step at $95^{\circ} \mathrm{C}$ for $10 \mathrm{~min}, 45$ cycles of denaturation at $95{ }^{\circ} \mathrm{C}$ for $10 \mathrm{~s}$, annealing for $20 \mathrm{~s}$, and extension at $72{ }^{\circ} \mathrm{C}$ for $15 \mathrm{~s}$. For HRM genotyping, the melting program included three steps: denaturation at 95 ${ }^{\circ} \mathrm{C}$ for $1 \mathrm{~min}$, renaturation at $40{ }^{\circ} \mathrm{C}$ for $1 \mathrm{~min}$, and a subsequent melting cycle with a continuous fluorescent reading from 65 to $90{ }^{\circ} \mathrm{C}$ at a rate of 25 acquisitions per degree celsius. Melting curve analysis was performed using the LightCycler 96 Gene Scanning Software (Roche, Germany) with default settings.

\section{PCR product sequencing and SNPs genotyping verifing}

PCR amplification using the above synthesized primers (Applied Biosystems GeneAmp PCR System 9700,USA). The reaction mixture contained 5-30 ng DNA, $2.5 \mu \mathrm{L}$ $10 \times$ Taq buffer, $0.5 \mu \mathrm{L}$ dNTP Mix, $0.25 \mu \mathrm{L}$ Taq DNA polymerase, $0.5 \mu \mathrm{L}$ each primer, $2 \mu \mathrm{L} \mathrm{MgCl}_{2}$, and adjusted with RNase-free water to a final volume of $25 \mu \mathrm{L}$. The amplification reaction was performed by denaturing at 95 ${ }^{\circ} \mathrm{C}$ for $10 \mathrm{~min}$; followed by 35 cycles of denaturing at 95 ${ }^{\circ} \mathrm{C}$ for $30 \mathrm{~s}$, annealing temperature for $30 \mathrm{~s}$, and extending at $72{ }^{\circ} \mathrm{C}$ for $45 \mathrm{~s}$ and a final extension at $72{ }^{\circ} \mathrm{C}$ for 20 min. The amplified product was sent to Shanghai Sangon Bioengineering Co., Ltd. (Shanghai, China) for cloning and sequencing, and the sequencing results were compared with the results of HRM genotyping to determine the SNP genotype. 
Table 3 SNP loci information used in the research

\begin{tabular}{|c|c|c|c|c|c|}
\hline Loci & Choromosome & SNP & Primer sequence $\left(5^{\prime}-3^{\prime}\right)$ & $\begin{array}{l}\text { Fragment } \\
\text { size (bp) }\end{array}$ & $\operatorname{Tm}\left({ }^{\circ} \mathrm{C}\right)$ \\
\hline 002 & 2 & $\mathrm{C} / \mathrm{T}$ & $\begin{array}{l}\text { F:AGAAAGACATAGGAGACCTC } \\
\text { R:GAAGCCTGGCTCTGATGTTC }\end{array}$ & 43 & 60 \\
\hline 003 & 4 & G/A & $\begin{array}{l}\text { F:GGTTCTAACTTACTGGGTTT } \\
\text { R:TGCTATCAGCAATGTTCTCA }\end{array}$ & 43 & 60 \\
\hline 004 & 9 & $\mathrm{~T} / \mathrm{C}$ & $\begin{array}{l}\text { F:GGAATGAAAGAACTTTGTC } \\
\text { R:GTCATTTATAGACTACAGAGTCA }\end{array}$ & 48 & 60 \\
\hline 008 & 5 & $\mathrm{~T} / \mathrm{G}$ & $\begin{array}{l}\text { F:TGTCTGCATCACATGGAGT } \\
\text { R:ATTGCTTTACCACATGCTC }\end{array}$ & 39 & 60 \\
\hline 014 & 6 & $\mathrm{~A} / \mathrm{G}$ & $\begin{array}{l}\text { F:GAGAAACCTGTAGTGTTTGG } \\
\text { R:GGTCCCTTTGATCTCATTC }\end{array}$ & 42 & 60 \\
\hline 015 & 19 & $\mathrm{~T} / \mathrm{C}$ & $\begin{array}{l}\text { F:AGCCAGATGGAAGGGAAGC } \\
\text { R:CGGTGACCAGGATGAAGC }\end{array}$ & 43 & 60 \\
\hline 017 & 9 & C/A & $\begin{array}{l}\text { F:CTTTCAGAAAGGCAGCAG } \\
\text { R:ACAGCTCTGTCTCCCTGG }\end{array}$ & 40 & 60 \\
\hline 020 & 1 & $\mathrm{~T} / \mathrm{C}$ & $\begin{array}{l}\text { F:CATTAGTACAGTAGTAACAGCTA } \\
\text { R:CATTGCTGTGAGCATTAGT }\end{array}$ & 48 & 58 \\
\hline 022 & 4 & $\mathrm{~A} / \mathrm{G}$ & $\begin{array}{l}\text { F:CTCCCAGGGGCTGAGCCT } \\
\text { R:CCTTCCTGACCCTATCCA }\end{array}$ & 39 & 58 \\
\hline 029 & 4 & $\mathrm{~A} / \mathrm{G}$ & $\begin{array}{l}\text { F:TGTCTGCTCCATGCTGTG } \\
\text { R:CAATCAGGATCTTGCCAT }\end{array}$ & 43 & 58 \\
\hline 039 & 2 & $\mathrm{C} / \mathrm{T}$ & $\begin{array}{l}\text { F:AGGGGGCTCCTTGTCCAT } \\
\text { R:GGTTTCCAGTGACTGATTAT }\end{array}$ & 43 & 60 \\
\hline 043 & 4 & G/A & $\begin{array}{l}\text { F:TCTTCTGCCCTTTTGTCA } \\
\text { R:GCAACTTTGGCATCTTTT }\end{array}$ & 45 & 60 \\
\hline 044 & 16 & $\mathrm{G} / \mathrm{T}$ & $\begin{array}{l}\text { F:ACTAGGATAAGACGGAAGC } \\
\text { R:ATCTTGCATTTTGCCTCC }\end{array}$ & 45 & 60 \\
\hline 046 & 1 & $\mathrm{C} / \mathrm{T}$ & $\begin{array}{l}\text { F:GGAATAGGGGTGCCAGGACAA } \\
\text { R:TGGAAATGAACTCTGACAAGG }\end{array}$ & 84 & 60 \\
\hline 051 & 5 & G/A & $\begin{array}{l}\text { F:GTTGTAGGGGCTAGGTATACTG } \\
\text { R:AATAGAAGATGGCGAGCCTG }\end{array}$ & 45 & 60 \\
\hline 053 & 7 & $\mathrm{C} / \mathrm{T}$ & $\begin{array}{l}\text { F:CAGAAATGTTGACAGAAA } \\
\text { R:TCATCTGGTATTTCACTT }\end{array}$ & 43 & 58 \\
\hline 061 & 13 & $\mathrm{C} / \mathrm{T}$ & $\begin{array}{l}\text { F:AGGTTCCATTATCACACACT } \\
\text { R:ACCTAGAGGCAGGTTTTG }\end{array}$ & 44 & 60 \\
\hline 063 & 3 & $\mathrm{C} / \mathrm{T}$ & $\begin{array}{l}\text { F:CAGCCTCACCTAATGTCC } \\
\text { R:GAGACTCATCTAGTTTCAA }\end{array}$ & 44 & 60 \\
\hline 064 & 3 & $\mathrm{~T} / \mathrm{C}$ & $\begin{array}{l}\text { F:GTACACCTGGTAGGACTCTGA } \\
\text { R:TGTGAAGATAGAGGAAATCTG }\end{array}$ & 48 & 60 \\
\hline 066 & 4 & C/A & $\begin{array}{l}\text { F:TCCTTGATGCTGACTCTT } \\
\text { R:TTATTCATACAGCATGTT }\end{array}$ & 37 & 60 \\
\hline 068 & 1 & $\mathrm{C} / \mathrm{T}$ & $\begin{array}{l}\text { F:TCCTCTGAGACAGGTGGGG } \\
\text { R:CTACACCTGCATCTCCATC }\end{array}$ & 40 & 60 \\
\hline 070 & 12 & $\mathrm{~T} / \mathrm{C}$ & $\begin{array}{l}\text { F:TTCTTAGAGGAAGGTTTAGT } \\
\text { R:CCATGCATTCTGATTTGAGG }\end{array}$ & 44 & 60 \\
\hline 074 & 13 & $\mathrm{~T} / \mathrm{C}$ & $\begin{array}{l}\text { F:TTTGCTTCCAGCCAGCC } \\
\text { R:GTGGAGAGCCCTAACCG }\end{array}$ & 39 & 60 \\
\hline 079 & 13 & $\mathrm{~A} / \mathrm{G}$ & $\begin{array}{l}\text { F:GGGATGGTAACAAGCGAAAT } \\
\text { R:TTCCATTGTTTAAGGAACCG }\end{array}$ & 45 & 60 \\
\hline 087 & 4 & G/A & $\begin{array}{l}\text { F:CAGCCTAAGCCCTGGAAT } \\
\text { R:TGCAAAACAGTTTGATGC }\end{array}$ & 43 & 60 \\
\hline
\end{tabular}


Table 3 (continued)

\begin{tabular}{|c|c|c|c|c|c|}
\hline Loci & Choromosome & SNP & Primer sequence $\left(5^{\prime}-3^{\prime}\right)$ & $\begin{array}{l}\text { Fragment } \\
\text { size (bp) }\end{array}$ & $\operatorname{Tm}\left({ }^{\circ} \mathrm{C}\right)$ \\
\hline \multirow[t]{2}{*}{088} & 4 & $\mathrm{~T} / \mathrm{C}$ & F:AAGCACAGAAAATAGCCA & 38 & 60 \\
\hline & & & R:GTGCAAAGCATCTCCCTC & & \\
\hline \multirow[t]{2}{*}{094} & 4 & $\mathrm{~T} / \mathrm{C}$ & F:CTAGGGTTCTAGGATGTC & 45 & 60 \\
\hline & & & R:ACAGGAACTGAGACTCAACT & & \\
\hline \multirow[t]{2}{*}{095} & 4 & $\mathrm{~A} / \mathrm{G}$ & F:CCCAACCACCAGAAGAGG & 39 & 60 \\
\hline & & & R:CTCCTCACAGAATCTGCA & & \\
\hline \multirow[t]{2}{*}{098} & 4 & $\mathrm{C} / \mathrm{T}$ & F:TTGGGGCAAGTTTCTGGG & 44 & 60 \\
\hline & & & R:CTGCCTACATCTAAATGACA & & \\
\hline \multirow[t]{2}{*}{103} & 4 & $\mathrm{~A} / \mathrm{G}$ & F:TAGACTCCGGGTGTAGCC & 39 & 60 \\
\hline & & & R:CACTCAATTCCTGAGCCA & & \\
\hline \multirow[t]{2}{*}{111} & 6 & $\mathrm{~T} / \mathrm{C}$ & F:GAACATTGTTCTGGATTTCA & 45 & 60 \\
\hline & & & R:TATCATGTCCCTGGGCTTGT & & \\
\hline \multirow[t]{2}{*}{112} & 4 & $\mathrm{G} / \mathrm{A}$ & F:GATAACTGGTTCTGTTCTCC & 43 & 60 \\
\hline & & & R:GTCGCAGCACAGTCAAGATG & & \\
\hline \multirow[t]{2}{*}{113} & 11 & $\mathrm{~T} / \mathrm{G}$ & F:GCAAGGGAGCATGGAAAC & 57 & 60 \\
\hline & & & R:TCCAAGACCGTCCGAAAT & & \\
\hline \multirow[t]{2}{*}{114} & 11 & $\mathrm{C} / \mathrm{T}$ & F:CTGCCCTCCAAGGTAAATCAC & 43 & 60 \\
\hline & & & R:GTATAGTCAAATCTTTCATCC & & \\
\hline \multirow[t]{2}{*}{115} & 11 & $\mathrm{~T} / \mathrm{C}$ & F:TCCTGGCTTCATTGTATTTTAC & 45 & 58 \\
\hline & & & R:AACATAAGTGCATTTTCCAC & & \\
\hline \multirow[t]{2}{*}{116} & 4 & $\mathrm{~T} / \mathrm{C}$ & F:TGAGGATGCACCAAGAAGC & 58 & 60 \\
\hline & & & R:ACATTGGAGTTAAGGGAGAAA & & \\
\hline \multirow[t]{2}{*}{124} & 2 & $\mathrm{~A} / \mathrm{G}$ & F:GCAGGTTTGCACTCTTACT & 47 & 60 \\
\hline & & & R:AAGCAACGAGTCATGTAAG & & \\
\hline
\end{tabular}

\section{Statistical analysis}

To test the effectiveness of the two methods, the parentage testing was performed by Cervus 3.0 software, which uses a maximum likelihood-based approach to infer parentage (Kalinowski et al. 2006). The heterozygosity and polymorphism information content of each allele from each sample was calculated at the same time. The sibling relationship was calculated using ML-Relate software (Kalinowski et al. 2007).

\section{Results and discussion}

Polymorphism analysis was carried out in 29 golden snubnosed monkey samples (including fecal samples) with 37 microsatellite markers. The results showed that the genotyping figures of D17S791 and D4S431 were disordered, D16S539 was not monomorphic, and GM213 and D6s287 were both homozygous. A total of 197 alleles were detected in the remaining 32 loci. The observed heterozygosity was $0.045-0.857$, the expected heterozygosity was $0.078-0.909$, the polymorphic information content was $0.074-0.883$, and the average polymorphic information content was 0.599
(Table 4). The amplification rate of blood samples and tissue samples was $100 \%$, and no zero allele was observed. The PCR effect of stool samples was relatively poor, and four loci (D11s925, D2s141, D6s287, D16S539) failed to amplify in this test.

The $12 \mathrm{blood} /$ tissue samples were subjected to HRM typing using 37 SNPs, and 29 loci were successfully genotyped, three were monomorphic $(008,068,124)$, and two were homozygous $(014,043)$. The observed heterozygosity was $0.083-0.583$, the expected heterozygosity was $0.083-0.518$, the polymorphic information content was $0.077-0.373$, and the average polymorphic information content was 0.244 with the remaining 24 loci (Table 5). To test the effect of this technology on stool samples, we amplified 046 with fecal DNA above. The dissolution curve failed to genotype by disorder, and the results of some samples failed to repeat. In this study, the CE-1P was 0.9582 , while the $\mathrm{CE}-2 \mathrm{P}$ was 0.7715 for the 24 SNPs (Fig. 1).

In the parentage testing for three offspring processed by 24 SNPs, there was one instance in which one's biological father was mistakenly referred to as his sibling in the process of parentage identification using the above 24 SNP loci. ML-Related software was used for further analysis. The software estimated the kinship between individuals using 
Table 4 Thirty-two microsatellite loci information of 29 samples

\begin{tabular}{|c|c|c|c|c|c|c|c|}
\hline Loci & $\begin{array}{l}\text { Allelic num- } \\
\text { bers }\end{array}$ & HObs & HExp & PIC & NE-1P & NE-2P & NE-PP \\
\hline GM206 & 13 & 0.500 & 0.909 & 0.883 & 0.356 & 0.216 & 0.073 \\
\hline D13s159 & 13 & 0.500 & 0.883 & 0.849 & 0.424 & 0.268 & 0.104 \\
\hline D20s206 & 8 & 0.609 & 0.850 & 0.810 & 0.508 & 0.336 & 0.162 \\
\hline GM105 & 8 & 0.625 & 0.833 & 0.791 & 0.538 & 0.364 & 0.185 \\
\hline D5s 1457 & 7 & 0.458 & 0.833 & 0.790 & 0.545 & 0.369 & 0.192 \\
\hline D17s1290 & 9 & 0.478 & 0.824 & 0.778 & 0.560 & 0.384 & 0.205 \\
\hline $\mathrm{D} 7 \mathrm{~s} 1830$ & 6 & 0.474 & 0.824 & 0.773 & 0.571 & 0.393 & 0.213 \\
\hline D1s533 & 8 & 0.615 & 0.811 & 0.768 & 0.570 & 0.393 & 0.208 \\
\hline D7s503 & 10 & 0.857 & 0.801 & 0.766 & 0.565 & 0.385 & 0.189 \\
\hline D14s306 & 7 & 0.640 & 0.800 & 0.754 & 0.589 & 0.411 & 0.223 \\
\hline D6s474 & 7 & 0.625 & 0.791 & 0.745 & 0.604 & 0.423 & 0.233 \\
\hline GM108 & 8 & 0.545 & 0.789 & 0.739 & 0.606 & 0.428 & 0.237 \\
\hline D7s2204 & 6 & 0.565 & 0.790 & 0.736 & 0.625 & 0.447 & 0.267 \\
\hline D6s493 & 6 & 0.565 & 0.768 & 0.711 & 0.651 & 0.474 & 0.290 \\
\hline D10s1432 & 6 & 0.826 & 0.767 & 0.711 & 0.650 & 0.473 & 0.288 \\
\hline D11s2002 & 5 & 0.522 & 0.770 & 0.710 & 0.657 & 0.481 & 0.302 \\
\hline GM109 & 5 & 0.346 & 0.707 & 0.639 & 0.731 & 0.563 & 0.389 \\
\hline D11s925 & 4 & 0.667 & 0.703 & 0.619 & 0.748 & 0.582 & 0.406 \\
\hline GM220 & 5 & 0.250 & 0.639 & 0.575 & 0.786 & 0.621 & 0.444 \\
\hline D15s644 & 9 & 0.400 & 0.602 & 0.569 & 0.786 & 0.600 & 0.387 \\
\hline D18s851 & 3 & 0.348 & 0.656 & 0.569 & 0.794 & 0.648 & 0.498 \\
\hline D7s1826 & 4 & 0.375 & 0.645 & 0.561 & 0.794 & 0.647 & 0.490 \\
\hline GM214 & 6 & 0.519 & 0.642 & 0.559 & 0.784 & 0.639 & 0.472 \\
\hline D16s420 & 5 & 0.174 & 0.557 & 0.481 & 0.845 & 0.707 & 0.553 \\
\hline D6s 271 & 6 & 0.160 & 0.495 & 0.460 & 0.869 & 0.705 & 0.525 \\
\hline GM227 & 5 & 0.440 & 0.534 & 0.452 & 0.858 & 0.734 & 0.591 \\
\hline D3s1768 & 3 & 0.269 & 0.461 & 0.366 & 0.898 & 0.808 & 0.703 \\
\hline GM228 & 4 & 0.045 & 0.289 & 0.267 & 0.959 & 0.850 & 0.737 \\
\hline GM209 & 4 & 0.150 & 0.276 & 0.256 & 0.963 & 0.856 & 0.744 \\
\hline D2s 141 & 2 & 0.167 & 0.290 & 0.239 & 0.961 & 0.880 & 0.803 \\
\hline D11s1366 & 3 & 0.091 & 0.172 & 0.160 & 0.986 & 0.916 & 0.847 \\
\hline GM219 & 2 & 0.080 & 0.078 & 0.074 & 0.997 & 0.963 & 0.930 \\
\hline
\end{tabular}

Fig. 1 The combined exclusion probability of the different number of microsatellite/SNP loci with one-parent-unknown/ known (CE-1P/CE-2P)

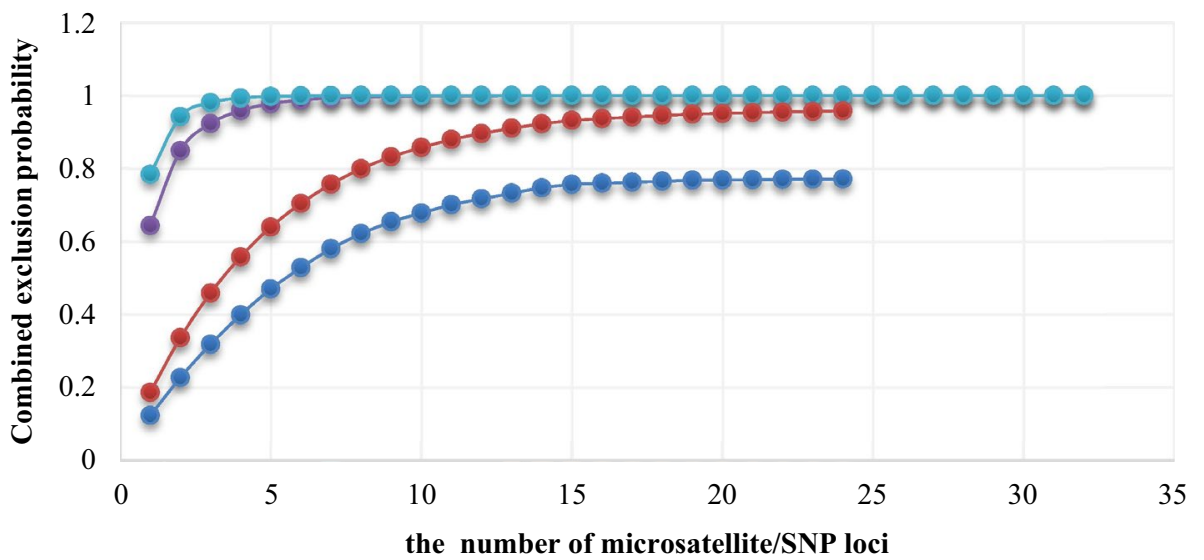

the number of microsatellite/SNP loci 
Table 5 Twenty-four SNP loci information of 12 golden monkey

\begin{tabular}{|c|c|c|c|c|c|c|c|}
\hline Loci & $\begin{array}{l}\text { Allelic num- } \\
\text { bers }\end{array}$ & HObs & HExp & PIC & NE-1P & NE-2P & NE-PP \\
\hline 002 & 2 & 0.083 & 0.083 & 0.077 & 0.997 & 0.962 & 0.928 \\
\hline 003 & 2 & 0.583 & 0.431 & 0.328 & 0.915 & 0.836 & 0.747 \\
\hline 015 & 2 & 0.167 & 0.159 & 0.141 & 0.988 & 0.929 & 0.874 \\
\hline 017 & 2 & 0.083 & 0.083 & 0.077 & 0.997 & 0.962 & 0.928 \\
\hline 020 & 2 & 0.583 & 0.489 & 0.359 & 0.890 & 0.821 & 0.729 \\
\hline 022 & 2 & 0.583 & 0.489 & 0.359 & 0.890 & 0.821 & 0.729 \\
\hline 029 & 2 & 0.500 & 0.464 & 0.346 & 0.901 & 0.827 & 0.737 \\
\hline 039 & 2 & 0.583 & 0.518 & 0.373 & 0.877 & 0.813 & 0.720 \\
\hline 046 & 2 & 0.250 & 0.344 & 0.275 & 0.946 & 0.862 & 0.779 \\
\hline 051 & 2 & 0.083 & 0.083 & 0.077 & 0.997 & 0.962 & 0.928 \\
\hline 061 & 2 & 0.083 & 0.083 & 0.077 & 0.997 & 0.962 & 0.928 \\
\hline 064 & 2 & 0.333 & 0.290 & 0.239 & 0.961 & 0.880 & 0.803 \\
\hline 079 & 2 & 0.500 & 0.391 & 0.305 & 0.930 & 0.848 & 0.761 \\
\hline 087 & 2 & 0.167 & 0.159 & 0.141 & 0.988 & 0.929 & 0.874 \\
\hline 088 & 2 & 0.167 & 0.159 & 0.141 & 0.988 & 0.929 & 0.874 \\
\hline 094 & 2 & 0.333 & 0.507 & 0.368 & 0.882 & 0.816 & 0.723 \\
\hline 095 & 2 & 0.333 & 0.507 & 0.368 & 0.882 & 0.816 & 0.723 \\
\hline 098 & 2 & 0.333 & 0.507 & 0.368 & 0.882 & 0.816 & 0.723 \\
\hline 103 & 2 & 0.500 & 0.391 & 0.305 & 0.930 & 0.848 & 0.761 \\
\hline 111 & 2 & 0.083 & 0.083 & 0.077 & 0.997 & 0.962 & 0.928 \\
\hline 112 & 2 & 0.167 & 0.159 & 0.141 & 0.988 & 0.929 & 0.874 \\
\hline 113 & 2 & 0.500 & 0.507 & 0.368 & 0.882 & 0.816 & 0.723 \\
\hline 114 & 2 & 0.417 & 0.344 & 0.275 & 0.946 & 0.862 & 0.779 \\
\hline 115 & 2 & 0.417 & 0.344 & 0.275 & 0.946 & 0.862 & 0.779 \\
\hline
\end{tabular}

the maximum likelihood method. According to the software results, the SNP marker operation was able to obtain an identification result consistent with the records after the sibling individual was excluded from the suspected sire list.

The criterion for the combined exclusion probability of non-single-parent was $\mathrm{PE}>0.9973$ in the international human parentage test. In this study, a minimum of five and up to nine microsatellite loci could meet the requirements of the standard if the dam was known or unknown. According to this study, CE-1P of nine loci reached 0.9977 (top 9 in Table 4), and CE-2P of five loci reached 0.9974 (top 5 in Table 4) (Fig. 1). However, the number of samples used in this study was low. When large-scale studies are carried out in the national captive population or wild population of the golden snub-nosed monkey, the combination may be changed.

The results of this study showed that the SNP loci did not meet the identification requirements. In order to understand the demands for SNP loci in the parentage testing of golden snub-nosed monkey, we doubled the 24 loci and calculated the 48 SNP loci using Cervus software. It was found that the combined exclusion probability of the no-parent reached 0.998 when the mother was known. However, the combined exclusion probability of the no-parent was 0.948 when the mother was unknown, which was still far from meeting the requirements. Therefore, in the follow-up study, more loci need to be developed for the SNP analysis.

A microsatellite locus can have multiple alleles, while a single SNP locus usually has only two alleles. Therefore, the polymorphism of a single microsatellite marker was higher than that of a SNP marker. The polymorphism of markers was the most important factor affecting the accuracy of parentage testing. The exclusion rate of loci was related to locus polymorphism: the lower the polymorphism, the smaller the exclusion rate (Zhang 2017). In order to meet the requirements for identification, it was necessary to increase the number of SNP loci (Liu et al. 2017; Zhang 2017). The study indicated that the number of SNP loci required was also related to the species population size of the estimated model. When the population was larger, the number of SNP loci required to meet the accuracy requirements of the identification was higher (Turakulov and Easteal 2003; Yu et al. 2015).

The results showed that the presence of siblings might lead to parentage identification errors in the absence of loci polymorphic information. Even though the parentage testing has been applied to several specific species, a large number of animals still do not have molecular markers or 
only a small number of molecular markers that have been developed. In this case, using ML-Related software to calculate the sibling/half sibling relationship and narrowing the suspected parentage range could be considered as an aid to improve accuracy, though it did not help improve the combined exclusion probability of the no-single-parent of the molecular markers.

Compared with the other sampling methods, fecal samples had the advantages of high availability as well as being non-invasive (Shan et al. 2018). However, fecal DNA is mainly obtained from intestinal exfoliated cells, so there were some problems such as low quantity, low quality, serious degradation, and low purity. Although the microsatellite amplification effect of the stool sample in this experiment was inferior to that of the blood sample, a large amount of data was still obtained. In the future, depth screening and optimization of experimental conditions could further improve the success rate, however, the SNP typing of fecal samples carried out by the HRM method could not accurately classify the dissolution curve in this study. This may be related to the loci we used, and does not necessarily represent the amplification ability of all SNP loci in feces. The results also showed the reading error of the HRM dissolution curve was greater than that of the gene sequencing for the fecal samples.

In this study, the accuracy of the two popular molecular markers was discussed in the parentage testing of golden snub-nosed monkey, and the results provided some reference significance for future research. Throughout the history of human parentage testing technology, as well as the laboratory's research experience in the field of parentage testing of endangered animals, such as giant pandas and red pandas, the establishment of a set of molecular markers has been a long-term process that requires patient and continuous development and screening. We expect more microsatellite markers and SNP markers to be developed and form a mature identification system in the future to provide greater resources for the conservation of golden snub-nosed monkey.

In conclusion, a small number of microsatellite loci (five to nine) with high polymorphism could be used to complete individual identification and parentage testing in this study. It was important to use microsatellite sites for individual identification and parentage testing because of the difficulty of sampling and the lack of samples of endangered species. However, there were some problems such as genetic variation and invalid alleles in microsatellite loci. There were some problems such as large samples and more loci were needed for identification, although SNP was more sensitive than microsatellite, and it has become a more favorable alternative marker in the world. In the future, researchers could choose a more suitable method for individual identification and parentage testing according to their own experimental conditions.

Acknowledgements We thank James Ayala for English editing. This work was supported by the Chengdu Research Base of Giant Panda Breeding.

\section{Compliance with ethical standards}

Conflict of interest The authors declare that they have no conflict of interests.

Open Access This article is licensed under a Creative Commons Attribution 4.0 International License, which permits use, sharing, adaptation, distribution and reproduction in any medium or format, as long as you give appropriate credit to the original author(s) and the source, provide a link to the Creative Commons licence, and indicate if changes were made. The images or other third party material in this article are included in the article's Creative Commons licence, unless indicated otherwise in a credit line to the material. If material is not included in the article's Creative Commons licence and your intended use is not permitted by statutory regulation or exceeds the permitted use, you will need to obtain permission directly from the copyright holder. To view a copy of this licence, visit http://creativecommons.org/licenses/by/4.0/.

\section{References}

Du LN (2015) Analysis of colobinae related to coat color genes and selection of SNPs in Rhinopithecus roxellana. M.A. Thesis. Guilin. Guangxi Normal University

Hao YL, Liu ZJ, Wu H, Ren BP, Wei FW, Li M (2007) Isolation and characterization of 11 microsatellite loci for the Sichuan snubnosed monkey, Rhinopithecus roxellana. Conserv Genet 8:10211024. https://doi.org/10.1007/s10592-006-9234-6

He G (2013) The role of kinship in the formation of golden monkey harem society. Ph.D. Dissertation. Xi'an: Northwest University

Heaton MP, Harhay GP, Bennett GL, Stone RT, Grosse WM, Casas E, Laegreid WW (2002) Selection and use of SNP markers for animal identification and paternity analysis in U.S. beef cattle. Mamm Genome 13:272-281. https://doi.org/10.1007/s0033 5-001-2146-3

Kalinowski ST, Wagner AP, Taper ML (2006) ML-relate: a computer program for maximum likelihood estimation of relatedness and relationship. Mol Ecol Notes 6:576-579. https://doi.org/10.111 1/j.1471-8286.2006.01256.x

Kalinowski ST, Wagner AP, Taper ML (2007) Revising how the computer program CERVUS accommodates genotyping error increases success in paternity assignment. Mol Ecol 16:10991106. https://doi.org/10.1111/j.1365-294X.2007.03089.x

Liu T, Li Q, Kong LF, Yu H (2017) Comparison of microsatellites and SNPs for pedigree analysis in the Pacific oyster Crassostrea gigas. Aquacult Int 25:1507-1519. https://doi.org/10.1007/s1049 9-017-0127-0

Pan D, Li Y, Hu HY, Meng SJ, Men ZM, Fu YX, Zhang YP (2005) Polymorphism analysis of the golden snub-nosed monkey based on microsatellite. Chin Sci Bull 50:2489-2494. https://doi. org/10.3321/j.issn:0023-074X.2005.22.009

Ren Y (2007) Parentage and genetic diversity studies in Sichuan snubnosed monkey (Rhinopithecus roxellana) using microsatellite DNA markers. M.A. Thesis. Xi'an: Northwest University 
Shan L, Hu YB, Wei FW (2018) Opportunities and challenges of fecal DNA technology in molecular ecology researches. Acta Theriologica Sinica 38:235-246

Turakulov R, Easteal S (2003) Number of SNPS loci needed to detect population structure. Hum Hered 55:37-45

Werner FAO, Durstewitz G, Habermann FA, Thaller G, Krämer W, Kollers S, Fries R (2004) Detection and characterization of SNPs useful for identity control and parentage testing in major European dairy breeds. Anim Genet 35:44-49. https://doi.org/10.104 6/j.1365-2052.2003.01071.x

Yu GC. Tang QZ, Long KR, Che TD, Li MZ, Shuai SR (2015) Effectiveness of microsatellite and single nucleotide polymorphism markers for parentage analysis in European domestic pigs. Genet Mol Res 14:1362-1370. https://doi.org/10.4238/2015.Febru ary. 13.15

Zhang YJ (2017) Phylogenetic analysis of ningxiang pig based on microsatellite DNA markers and SNP chip. M.A. Thesis. Changsha: Hunan Agricultural University

Zhang ZH, Shen FJ, Sun S, David VA, Zhang AJ, O'Brien J S (2003) Paternity assignment of giant panda by microsatellite genotyping.
Hereditas 25(5):504-510. https://doi.org/10.1016/S0891 -0618(02)00103-5

Zhang WP, Zhang ZH, Wei K, Shen FJ, Hou R, Zhang L, Yue BS (2006) Isolation and characterization of polymorphic tri- and tetra- nucleotide microsatellite loci for the south China tiger. J Nat Hist 40:2259-2263. https://doi.org/10.1080/00222930601076110

Zhang L, Zhang ZH, Shen FJ, Hou R, Zhang WP, Liu YL, Yang AL (2015) Identification and characterization of polymorphic microsatellite loci in the red-crowned crane. Genet Mol Res 14:1516915176. https://doi.org/10.4238/2015.November.25.5

Zhou YY, Yang WY, Zhang YG, Lu H, Liu X, Li J, Feng JC (2015) Genetic diversity and relatedness of the golden snub-nosed monkey provisioned group in Shennongjia National Natural Reserve. Acta Theriologica Sinica 35:229-240

Publisher's Note Springer Nature remains neutral with regard to jurisdictional claims in published maps and institutional affiliations. 